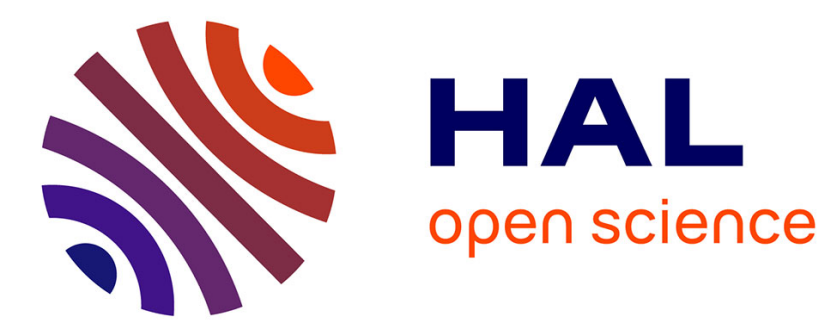

\title{
Appareillage de mesure de densité au 1/10 000e Application à l'étude des défauts dans le séléniure de plomb
}

\author{
C. Belin, R. Pellegrin
}

\section{To cite this version:}

C. Belin, R. Pellegrin. Appareillage de mesure de densité au 1/10 000e Application à l'étude des défauts dans le séléniure de plomb. Revue de Physique Appliquée, 1971, 6 (3), pp.415-418. 10.1051/rphysap:0197100603041500 . jpa-00243564

\section{HAL Id: jpa-00243564 https://hal.science/jpa-00243564}

Submitted on 1 Jan 1971

HAL is a multi-disciplinary open access archive for the deposit and dissemination of scientific research documents, whether they are published or not. The documents may come from teaching and research institutions in France or abroad, or from public or private research centers.
L'archive ouverte pluridisciplinaire HAL, est destinée au dépôt et à la diffusion de documents scientifiques de niveau recherche, publiés ou non, émanant des établissements d'enseignement et de recherche français ou étrangers, des laboratoires publics ou privés. 


\title{
APPAREILLAGE DE MESURE DE DENSITÉ AU 1/10 000" APPLICATION A L'ÉTUDE DES DÉFAUTS DANS LE SÉLÉNIURE DE PLOMB
}

\author{
C. BELIN et R. PELLEGRIN \\ Laboratoire de Magnétisme et de Physique des Solides, C. N. R. S., 92, Meudon-Bellevue \\ (Reçu le 11 juillet 1971)
}

\begin{abstract}
Résumé. - Un appareillage de mesure de densité des solides, utilisant les pesées hydrostatiques, a été mis au point. La régulation de température du liquide d'immersion permet d'obtenir une précision de $\pm 3 \times 10^{-3}{ }^{\circ} \mathrm{C}$. La méthode a été appliquée à l'étude des défauts de $\mathrm{PbSe}$ et a permis de conclure à l'existence de lacunes de $\mathrm{Pb}$ dans les échantillons de type $p$.
\end{abstract}

Abstract. - An apparatus for making density measurements of solids by hydrostatic weighing is described. The temperature of the immersion liquid can be regulated to within $\pm 3 \times 10^{-3}{ }^{\circ} \mathrm{C}$. This method was used to study the defects of $\mathrm{PbSe}$ and the presence of $\mathrm{Pb}$ vacancies in $p$-type samples was ascertained.

1. Introduction. - De nombreux composés binaires présentent des écarts à la stœchiométrie qui peuvent être très importants : par exemple jusqu'à $20 \%$ dans $\mathrm{FeO}$ [1]. Les mesures de densité sont un des moyens possibles pour étudier ces écarts et caractériser les défauts ponctuels qu'ils impliquent à l'échelle atomique (cf. par exemple [2]). Dans les semiconducteurs, en particulier, des concentrations mêmes faibles $\left(10^{14}\right.$ à $\left.10^{21} \mathrm{~cm}^{-3}\right)$ de ces défauts peuvent influencer les propriétés électriques et optiques : d'où le besoin de mesures de densité d'une précision au moins égale à $10^{-5}$ en valeur relative. Une comparasson critique des méthodes existantes nous a conduits à adopter celle qui fait appel aux pesées hydrostatiques [3].

Après avoir décrit l'appareillage que nous avons construit, nous donnerons les résultats de mesures sur le séléniure de plomb contenant un excès de sélénium, ce qui permettra de discuter concrètement la précision qu'il est possible d'atteindre avec nos conditions expérimentales.

2. Description de l'appareillage de mesure. 2.1 LA MiCROBALANCE. - Nous avons utilisé une microbalance Mettler M-5 de portée $20 \mathrm{~g}$, dont la précision peut être de $\pm 1 \mu \mathrm{g}$. Afin de disposer, pour effectuer les pesées, d'un espace plus vaste que celui prévu généralement à cet effet, nous avons placé la balance sur un trépied d'une vingtaine de centimètres de hauteur et percé le fond d'un orifice de petit diamètre qui permet le passage d'une tige métallique, solidaire du plateau de pesée et terminée par un crochet (Fig. 1a). L'ensemble repose, par l'intermédiaire d'une dalle en béton et de quatre amortisseurs, sur un châssis fixé au sol et dont les montants traversent, sans contact, une table sur laquelle est placée une boîte à gants protégeant la balance de courants d'air éventuels.

La pièce dans laquelle est installé ce montage est

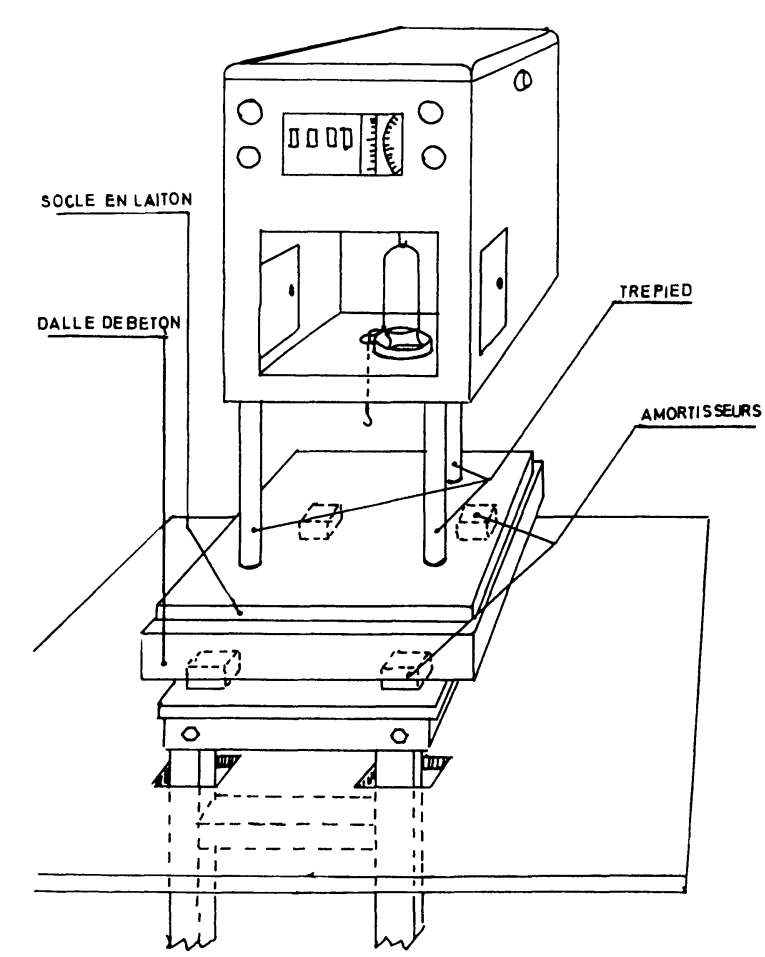

FIG. 1a. - Schéma du dispositif support de balance et balance.

climatisée de telle sorte que les variations de la température soient de $\pm 1{ }^{\circ} \mathrm{C}$ à $25^{\circ} \mathrm{C}$. Dans la boîte à gants qui, tout à la fois, isole la balance de l'extérieur et joue le rôle d'écran thermique, ces fluctuations n'excèdent pas le demi-degré.

\subsection{DisPositif DE RÉGULATION DE LA TEMPÉRATURE} DU LIQUIDE D'IMMERSION. - Les variations importantes de la densité de l'eau en fonction de la température nous ont conduits à utiliser une enceinte thermostatée permettant d'opérer à température constante.

a) Description de l'enceinte. - Cette enceinte: construite par la Société Alcatel (Fig. 1b), est constituée par un récipient en cuivre, revêtu intérieurement 
d'une mince pellicule de téflon dont la présence n'est justifiée que par l'utilisation possible de liquides très corrosifs. L'ensemble se trouve isolé thermiquement des influences extérieures par un revêtement en mousse de poly-uréthane. Un couvercle, dans lequel a été ménagé un orifice pour le passage des échantillons, vient se placer à la partie supérieure.

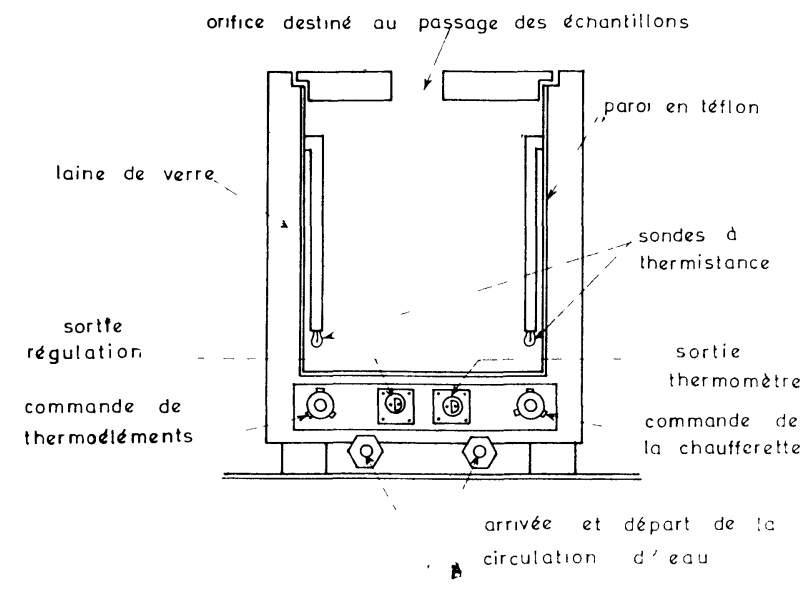

FIG. 1b. - Schéma de l'enceinte thermostatée.

Cette enceinte est équipée, à sa base, de deux modules thermoélectriques et d'une circulation d'eau, d'un débit d'environ $1 \mathrm{l} / \mathrm{mn}$ qui permet l'élimination des calories absorbées par les thermoéléments au sein du liquide. Deux sondes à thermistance plongeant dans le liquide sont destinées, l'une à la régulation et l'autre au contrôle de sa température.

b) Principe de fonctionnement. - La thermistance de régulation $R_{\mathrm{th}}$ et un potentiomètre de réglage sont intercalés dans les branches d'un pont de Wheatstone. Afin que la sensibilité de ce dernier soit la plus grande possible, il serait nécessaire de l'alimenter sous une tension $V$ élevée. Compte tenu du pouvoir maximum de dissipation de la thermistance, nous avons adopté les valeurs suivantes :

$$
V=15 \mathrm{~V} \quad \text { et } \quad R_{\mathrm{th}}=100 \mathrm{k} \Omega .
$$

La tension d'erreur apparue aux bornes du pont est amplifiée et commande, suivant sa polarité, les amplificateurs de puissance des voies de chauffage et de refroidissement.

Cependant les deux amplificateurs de puissance possèdent un seuil (Fig. 2); il est nécessaire, avant de les attaquer, d'utiliser un circuit dont la fonction de transfert en tension est schématisée par la figure 3. L'ensemble du circuit de régulation est décrit par la figure 4.

c) Résultats obtenus. - Le revêtement interne de l'enceinte par du téflon, mauvais conducteur thermique, introduit une constante de temps qui limite les performances de la régulation. Cependant, les fluctuations enregistrées ne dépassent pas, en régime stable, $3 \times 10^{-4}{ }^{\circ} \mathrm{C}$. Il est remarquable de constater que ce résultat peut être obtenu en un temps très

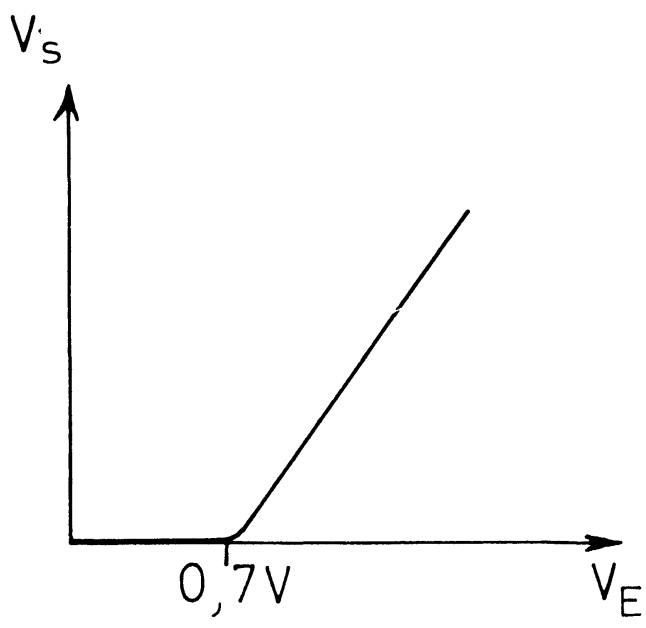

Fig. 2. - Tension seuil $\left(V_{\mathrm{S}}\right)$ en fonction de la tension erreur $\left(V_{\mathrm{E}}\right)$ des amplificateurs de puissance.

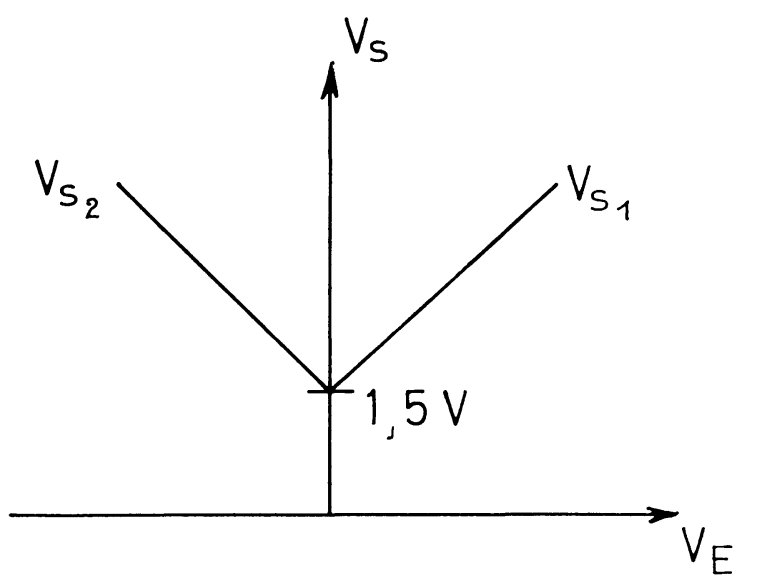

FIG. 3. - Schéma de la fonction de transfert de tension du circuit permettant l'attaque des amplificateurs de puissance.

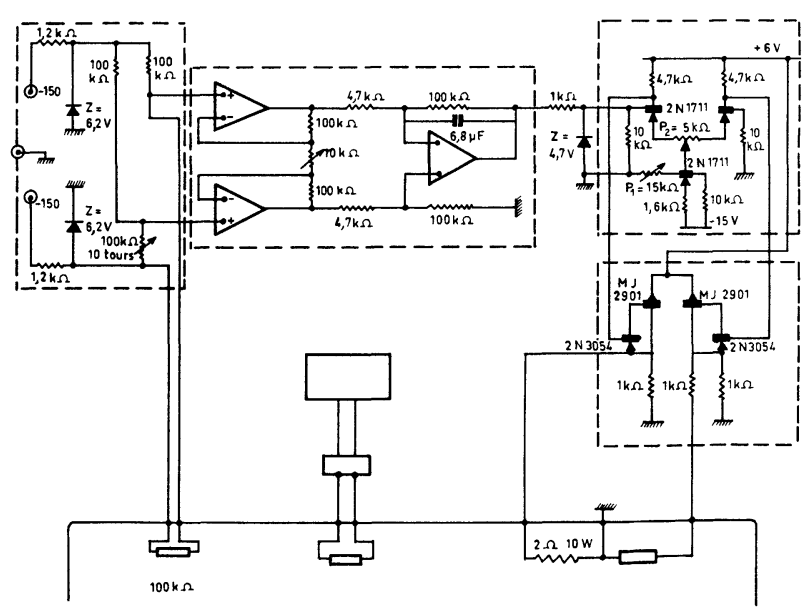

Fig. 4. - Schéma de l'ensemble du circuit de régulation.

court. En effet, il faut environ $30 \mathrm{mn}$ pour stabiliser à $25^{\circ} \mathrm{C}$ un liquide dont la température initiale est de $20^{\circ} \mathrm{C}$ et quelques minutes pour compenser les perturbations occasionnées par l'immersion d'un échantillon dont la température diffère de celle du liquide de quelques dixièmes de degrés. 
Les modules thermoélectriques que nous avons utilisés et qui se révèlent d'un emploi très aisé contribuent pour beaucoup à la qualité de la régulation, notamment en ce qui concerne les temps d'établissement de l'équilibre.

Il est à noter également que cette enceinte fonctionne depuis près de deux ans sans avoir jamais présenté la moindre défaillance.

3. Principe des mesures et processus expérimental. Le principe de la méthode de détermination des densités par pesées hydrostatiques est relativement simple, puisqu'il suffit de deux pesées effectuées dans deux fluides de densités différentes pour obtenir la masse et le volume d'un échantillon. La première se pratiquera dans l'air et la seconde dans un liquide, par exemple dans l'eau.

Si nous appelons $M$ la masse de l'échantillon, $V$ son volume et $M^{\prime}$ et $M^{\prime \prime}$ ses masses apparentes dans l'air, et dans l'eau, nous aurons successivement:

- dans l'air : $M^{\prime}=M-V d_{\mathrm{air}}\left(d_{\mathrm{air}}\right.$ est la densité de l'air) ;

- dans l'eau : $M^{\prime \prime}=M-V d_{\text {eau }}$ ( $d_{\text {eau }}$ est la densité de l'eau), et par suite la densité $d$ de l'échantillon est égale à :

$$
d=\frac{M^{\prime} d_{\mathrm{eau}}-M^{\prime \prime} d_{\mathrm{air}}}{M^{\prime}-M^{\prime \prime}}
$$

Les pesées dans l'air, effectuées à l'intérieur de la boîte à gants, n'ont pas soulevé de difficultés, contrairement à celles qui ont été réalisées dans l'eau. En effet, si les variations de la température du liquide, ont pu être réduites par l'utilisation de l'enceinte décrite précédemment, nous nous sommes heurtés aux inconvénients provenant des effets de la tension superficielle sur le fil soutenant l'échantillon.

En raison de la présence d'inhomogénéités locales, la surface du fil et celle de l'eau ne se raccordent pas de façon identique d'une pesée à l'autre. Un nettoyage au mélange sulfo-chromique bouillant s'étant avéré inefficace, nous avons utilisé le fait qu'un liquide peut mouiller parfaitement un solide si celui-ci présente à sa surface des aspérités microscopiques. Pour cela, nous avons réalisé un dépôt électrolytique de platine qui nous a permis d'obtenir une surface poreuse parfaitement homogène. Si des observations, faites à l'aide d'une lunette binoculaire, nous confirmèrent que la surface du liquide venait se raccorder tangentiellement à celle du fil, la reproductibilité des

\begin{tabular}{ccc}
$\begin{array}{c}\text { Echantillon } \\
\text { de } \mathrm{PbSe}\end{array}$ & \multicolumn{2}{c}{$\mathrm{T}\left({ }^{\circ} \mathrm{K}\right)$ de l'ampoule } \\
- & $\begin{array}{c}\text { échantillon } \\
\text { point froid }\end{array}$ & - \\
$\mathrm{n}^{\circ} 1$ & 1000 & 630 \\
$\mathrm{n}^{\circ} 2$ & 1000 & 710 \\
$\mathrm{n}^{\circ} 3$ & 1000 & 790 \\
$\mathrm{n}^{\circ} 4$ & 1000 & 890
\end{tabular}

mesures ne s'en trouva que faiblement améliorée. Finalement, ce n'est qu'en utilisant un agent tensioactif, le sulfate-lauryl de sodium, que nous avons pu obtenir des résultats reproductibles.

Le remplacement de l'eau par un liquide organique lourd pourrait en principe conduire à une meilleure précision. Cependant nous n'avons pu éviter, avec un tel liquide, divers inconvénients : émission de vapeurs corrosives, lente décomposition faisant évoluer la densité dans le temps, forte tension superficielle. L'immersion dans l'eau s'est avérée suffisante pour étudier les défauts de réseau de $\mathrm{PbSe}$.

Compte tenu du processus expérimental adopté, les mesures de densité sont obtenues avec une précision relative de $2 \times 10^{-4} \mathrm{~g} / \mathrm{cm}^{3}$ [3].

4. Etude des défauts dans PbSe. - Nous avons utilisé, pour nos mesures de densité, des échantillons monocristallins ou parfois bicristallins, d'environ $1 \mathrm{~cm}^{3}$ et convenablement décapés.

Nos premières mesures ont porté sur deux séries d'échantillons, les uns de type $p$, les autres de type $n$, tous possédant une forte concentration de défauts, obtenus par des traitements de recuit appropriés [4].

Nous avons obtenu les résultats suivants :

PbSe type $p: p=7 \times 10^{18} \mathrm{~cm}^{-3}$;

$$
d=8,3085 \pm 2 \times 10^{-4} \mathrm{~g} / \mathrm{cm}^{3} \text {. }
$$

PbSe type $n: n=4 \times 10^{18} \mathrm{~cm}^{-3}$;

$$
d=8,3125 \pm 2 \times 10^{-4} \mathrm{~g} / \mathrm{cm}^{3} \text {. }
$$

Ces deux seules valeurs ne nous permettent pas de conclure quant à la nature des défauts présents. Elles laissent cependant prévoir, du fait de leur écart relativement important, que des mesures effectuées sur des échantillons d'un type unique, mais présentant des concentrations en porteurs variables, nous le permettront. Nous avons utilisé pour ces mesures des cristaux recuits en ampoule scellée sous tension de vapeur contrôlée [5].

Nous avons porté sur un même graphique, d'une part la courbe représentative de la variation de la densité expérimentale en fonction de la concentration en porteurs, et d'autre part, les courbes des variations de la densité théorique, calculées dans l'hypothèse des différents types de défauts, supposés une fois ionisés (Fig. 5). Nous pouvons constater que la pente de la courbe expérimentale s'ajuste à celle de la courbe théorique tracée dans l'hypothèse où les lacunes de plomb prédominent.

$\begin{array}{ccc}\begin{array}{c}\text { Pression } \\ \text { de } \mathrm{Se}_{2} \\ (\mathrm{~mm} \mathrm{de} \mathrm{Hg})\end{array} & \begin{array}{c}\text { Concentration } \\ \text { de trous } \\ \left(\mathrm{cm}^{-3}\right)\end{array} & \begin{array}{c}d \\ \left(\mathrm{~g} / \mathrm{cm}^{-3}\right)\end{array} \\ - & - & - \\ 1 & 3,8 \times 10^{18} & 8,3089 \\ 10 & 7,6 \times 10^{18} & 8,3074 \\ 100 & 1,4 \times 10^{19} & 8,3060 \\ 320 & 2,9 \times 10^{19} & 8,3051\end{array}$




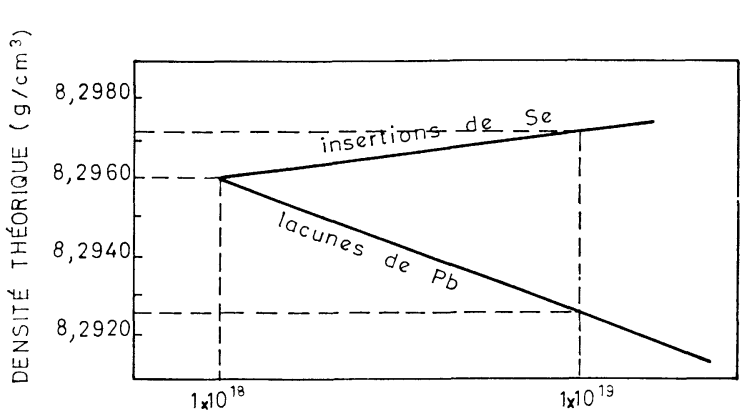

(a)

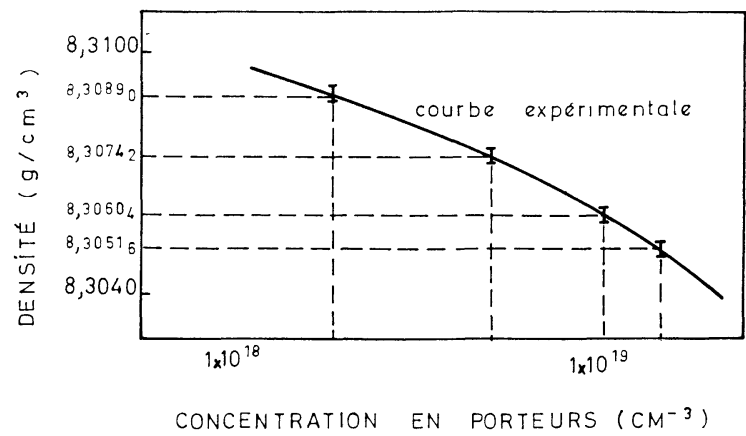

(b)

FIg. 5. - Courbes théorique et expérimentale de la densité de $\mathrm{PbSe}$ contenant un excès de sélénium.

Nous pouvons donc conclure que les défauts provenant d'un excès de sélénium dans les cristaux de séléniure de plomb de type $\mathrm{p}$ sont des lacunes de plomb; ce résultat est en accord avec celui de H. Gobrecht et A. Richter [6].

Des mesures fines de paramètre de réseau, réalisées sur des échantillons des deux types, viennent confirmer ce résultat. Ces déterminations ont été effectuées par diffraction $\mathrm{X}$ selon la méthode de Debye-Scherrer, en utilisant le rayonnement $K_{\alpha_{1}}$ du cuivre, dont la longueur d'onde est : $\gamma=1,540562 \pm 8 \times 10^{-6} \AA$ [7]. Sur le diagramme complet de diffraction, nous avons ainsi une raie particulière, toujours la même pour tous les échantillons étudiés. Dans le cas de l'échantillon de type $n$, nous constatons qu'il lui correspond l'angle $2 \theta=140,36^{\circ}$. Par contre, quel que soit celui de type $p$, cet angle a pour valeur : $20=140,39^{\circ}$. Nous pouvons donc constater que les lacunes de plomb ne perturbent pas sensiblement le réseau.
Ceci justifie l'hypothèse faite dans le calcul de variation de la densité théorique, que le volume de la lacune de plomb est sensiblement égal au volume ionique du plomb.

Une détermination plus exacte, effectuée de 1/100 de degré en $1 / 100$ de degré à partir de la valeur $2 \theta=140^{\circ}$, nous a donné les valeurs suivantes :

PbSe type $p: 2 \theta=140,390^{\circ} \pm 0,005^{\circ}$

PbSe type $n: 2 \theta=140,367^{\circ} \pm 0,005^{\circ}$.

Les distances réticulaires $d_{\mathrm{nk} 1}$ et les paramètres $a$ correspondants sont :

PbSe type $p: d_{\mathrm{nk} 1}=0,818707 \AA \quad a_{\mathrm{p}}=6,1266 \AA$ PbSe type $n: d_{\mathrm{nkl}}=0,818768 \AA \quad a_{\mathrm{n}}=6,1271 \AA$.

L'erreur sur le paramètre s'exprime par :

$$
\frac{\Delta a}{a}=\frac{\Delta d_{\mathrm{nk} 1}}{d_{\mathrm{nk} 1}}=\frac{\Delta \lambda}{\lambda}+\frac{\Delta \theta(\mathrm{rad})}{\operatorname{tg} \theta}
$$

ce qui donne :

$$
\Delta a \simeq 2 \times 10^{-4} \AA .
$$

La différence des paramètres de réseau de $\mathrm{PbSe}$ de type $p$ et $n$ est donc faible, mais significative. Le fait que le paramètre de réseau et la densité des échantillons de type $n$ aient des valeurs supérieures à celles obtenues pour le type $p$ tendrait à prouver, en accord avec différents auteurs qui ont étudié la diffusion du ${ }^{210} \mathrm{~Pb}$ et $\mathrm{du}{ }^{75} \mathrm{Se}[8]$ que l'excès de $\mathrm{Pb}$ dans ces échantillons se traduit par l'existence d'atomes interstitiels de plomb.

5. Conclusion. - Nous avons réalisé un appareillage destiné à la mesure des densités par une méthode de pesées hydrostatiques et permettant de déterminer la nature de certains défauts simples existant au sein d'un réseau cristallin. A la limite de sensibilité de l'appareillage, il est possible d'apprécier des différences de concentration de défauts ponctuels inférieures à $10^{18} \mathrm{~cm}^{-3}$, pour un poids atomique égal à celui du plomb, et de l'ordre de $2 \times 10^{18} \mathrm{~cm}^{-3}$ pour le sélénium.

Remerciements. - Nous exprimons notre gratitude à Mme $\mathrm{H}$. Rodot qui nous a donné ce sujet de recherches et qui nous a fait profiter de son expérience ; nous remercions aussi $\mathbf{J}$. Rioux à qui nous devons les mesures radiocristallographiques.

\section{Bibliographie}

[1] Benard (J.), Bull. Soc. Chim., 1949, 16, D 109.

[2] Huber (M.), J. Physique, 1963, 24, 438.

[3] Beurn (C.), Thèse de 3e Cycle, Paris, 1969.

[4] Brebrick (R.), Gubner (E.), J. Chem. Phys., 1962, 36, 170.

[5] Ohashi (N.), Igaki (K.), Bull. Univ. Osaka Prefecture 1962, A 11, no $1,89$.
[6] Gobrecht (H.), Richter (A.), J. Physique, 1965, 26, 1839.

[7] Bearden (J. A.), U. S. Energy Commission Division of Technical Informations Extension Dakridge, Tennessee, 1964.

[8] Seltzer (M. S.), Wagner (J. B.), J. Chem. Phys., 1962, 36,130 ;

Boltaks (B. I.), Mokhov (I.), Soviet Phys. Techn. Phys., 1959, 3, 5. 